\title{
Superconductivity in tin selenide under pressure
}

\author{
Giovanni Marini $\odot,,^{1,2}$ Paolo Barone $\odot,^{2}$ Antonio Sanna $\odot,{ }^{3}$ Cesare Tresca $\odot,{ }^{4,5}$ Lara Benfatto $\odot,{ }^{6}$ and Gianni Profeta $\odot^{1,2}$ \\ ${ }^{1}$ Department of Physical and Chemical Sciences, University of L'Aquila, Via Vetoio 10, I-67100 L'Aquila, Italy \\ ${ }^{2}$ SPIN-CNR, University of L'Aquila, Via Vetoio 10, I-67100 L'Aquila, Italy \\ ${ }^{3}$ Max-Planck Institut für MikrostrukturPhysik, Weinberg 2, 06120 Halle, Germany \\ ${ }^{4}$ Department of Physics, La Sapienza University of Rome, Piazzale A. Moro 5, 00185, Rome, Italy \\ ${ }^{5}$ Institut des Nanosciences de Paris, Sorbonne Universités-UPMC Universite Paris 6 \\ and CNRS-UMR 7588, 4 place Jussieu, F-75252 Paris, France \\ ${ }^{6}$ ISC-CNR and Department of Physics, Sapienza University of Rome, Piazzale A. Moro 5, 00185, Rome, Italy
}

(Received 30 July 2019; revised manuscript received 20 September 2019; published 14 November 2019)

\begin{abstract}
Tin selenide is a layered material that captured the interest of the scientific community for its stunning thermoelectric properties and fascinating phase transitions under pressure. Recently, an experimental study revealed the existence of a topological and superconducting phase in its pressure-stabilized CsCl-type phase. By means of ab initio techniques, we investigate the structural properties of this compound and its pressure phase diagram, comparing our findings with the experimental results. We then focused on the electronic features of the topological CsCl-type phase and analyze its dynamical and superconducting properties. To understand the origin of the superconducting transition, we predict the critical temperature as a function of the pressure, $T_{c}(P)$, by the superconducting density-functional theory and analyze the behavior of the resistance with pressure and temperature by means of a percolative model. The careful comparison of calculations with available experiments reveals that inhomogeneities and nonhydrostatic pressure effects are relevant in this system.
\end{abstract}

DOI: 10.1103/PhysRevMaterials.3.114803

\section{INTRODUCTION}

Tin selenide, SnSe, is a IV-VI binary narrow-gap semiconducting compound that has recently been subject to extensive study because of its fascinating physical properties. In the last years, its potential as a highly efficient thermoelectric material has been investigated in depth after the report of its strikingly thermoelectric figure of merit $(\mathrm{ZT} \simeq 2.6$ at $923 \mathrm{~K})$, stemming from the combination of a high power factor and low thermal conductivity due to strong anharmonic effects [1-4].

At ambient conditions, SnSe crystallizes in the orthorhombic GeS-type structure sketched in Fig. 1(a) (space group Pnma) [5], that can be viewed as a derivative of the black phosphorus structure [6,7]. In the quest for phosphorene analogues, SnSe and isostructural group-IV monochalcogenides $\mathrm{SnS}, \mathrm{GeS}$, and GeSe have been recently studied, unveiling their multiple-valley electronic structures, whose valley separation is enhanced by the reduction of dimensionality, being at the same time addressable either with linearly polarized light or transverse nonlinear conductivity [6-8]. Furthermore, it has been predicted that $\mathrm{SnSe}$ may display a topological crystalline insulating phase [9], analogous to that observed in rock-salt SnTe $[10,11]$ when stabilized in the cubic NaCl-type structure [12] [space group $F m \overline{3} m$, shown in Fig. 1(e)]. Extensive research has been done on the topological properties of IV (Ge, $\mathrm{Sn}, \mathrm{Pb}$ )-VI ( $\mathrm{S}, \mathrm{Se}, \mathrm{Te})$ semiconducting monochalcogenides, both in their bulk and monolayer forms and addressing, just to mention a few, the effects of pressure, strain, alloying, and lattice dynamics [13-18]. For example, it was reported that the cubic $F m \overline{3} m$ structure can be experimentally achieved, e.g., by growing $\mathrm{SnSe}$ epitaxially on a $\mathrm{NaCl}$ substrate [19].
Indeed, the structural and electronic properties of $\mathrm{SnSe}$ are even richer under high pressure conditions. SnSe undergoes two pressure-induced structural transitions: the first one, from orthorhombic Pnma GeS-type to orthorhombic $\mathrm{Cmcm}$ structure, shown in Fig. 1(d), has been reported to occur at $10.5 \mathrm{GPa}$ [21]; the second, from orthorhombic $\mathrm{Cmcm}$ to cubic CsCl-type structure, shown in Fig. 1(c) (space group $P m \overline{3} m$ ) has been recently observed at approximately $27 \mathrm{GPa}$ [22], in contradiction with a previous work not reporting the transition up to $34 \mathrm{GPa}$ [23]. Moreover, another orthorhombic Pnma phase, dynamically coexisting with Pnma GeS-type and $\mathrm{Cmcm}$ phases, has been recently proposed in the 0-5 GPa range [24]. This phase, that will be referred to as $\gamma$-Pnma in the following, is a distorted GeS-type structure [as shown in Fig. 1(b)] that has also been observed in the pressure phase diagram of SnTe in the range 5-18.3 GPa [25]. Overall, a nontrivial dynamic phase coexistence of closely related structures has been proposed in the pressure range 1-13.4 GPa at room temperature, supported by x-ray diffraction (XRD) measurements [24]. Finally, a phase with the unconventional stoichiometry $\mathrm{Sn}_{3} \mathrm{Se}_{4}$ (space group $I \overline{4} 3 d$ ) has been synthesized in laser-heated diamond anvil cell around $16 \mathrm{GPa}$ [26].

As expected, pressure also affects electronic properties, inducing a semiconductor-metal transition around $12 \mathrm{GPa}$, close to the first structural transition between the orthorhombic GeS-type and $\mathrm{Cmcm}$ phases [5,22]. More interestingly, a superconducting (SC) phase has been reported at higher pressures since 1997 [27] and recently confirmed by Chen and collaborators in Ref. [22]. However, even if both reports agree in identifying the CsCl-type structure as the SC phase, there is no agreement neither on the pressure range at which 

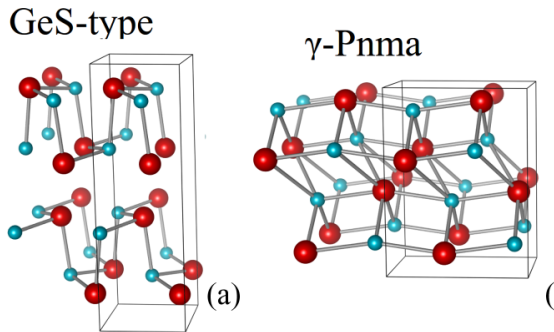

(b)
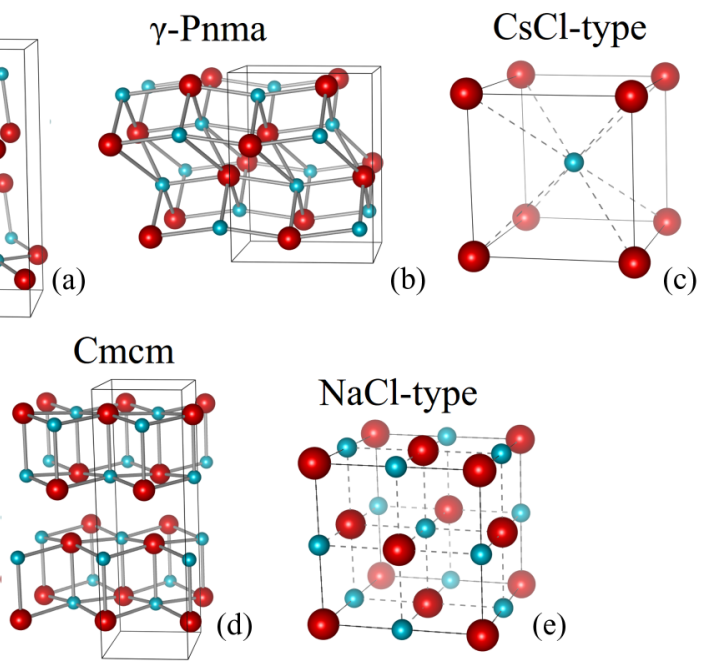

FIG. 1. Pnma GeS-type [panel (a)], $\gamma$-Pnma [panel (b)], CsCltype [panel (c)], Cmcm [panel (d)], and $\mathrm{NaCl}$-type [panel (e)] structures [20]. Red and blue atoms are $\mathrm{Sn}$ and Se, respectively.

superconductivity develops nor on the transition temperature as a function of the pressure, $T_{c}(P)$ : in Ref. [27], the authors find a superconducting $T_{c} \simeq 4.5 \mathrm{~K}$ at $58 \mathrm{GPa}$, while in Ref. [22] the SC phase starts at $\simeq 27 \mathrm{GPa}$, when the second structural phase transition between the orthorhombic $\mathrm{Cmcm}$ and the cubic CsCl-type structures takes place, with a critical temperature $T_{c} \simeq 3 \mathrm{~K}$ extending up to $\simeq 55 \mathrm{GPa}$ [22]. XRD measurements suggest a coexistence of orthorhombic $\mathrm{Cmcm}$ and cubic CsCl-type structures up to $\simeq 43.4 \mathrm{GPa}$, where $T_{c}$ takes its maximum value [22]. Theoretical predictions revealed interesting topological features in the metallic $\mathrm{CsCl}-$ type phase of SnSe [22], suggesting the presence of nontrivial surface states protected by $Z_{2}$ topology and weakly dispersing along selected directions that might affect SC properties [28]. Along the same research line, sulfur doping has been shown to enhance the SC properties of SnSe, bringing the $T_{c}$ over $4 \mathrm{~K}$ and extending the pressure range of superconductivity beyond $70 \mathrm{GPa}$ [29]. Superconducting phases, with enhanced critical temperatures up to $6 \mathrm{~K}$, have been also reported in doped (IV, III)-VI compounds, like $\mathrm{Sn}_{1-x} \mathrm{In}_{x} \mathrm{Te}_{1-y} \mathrm{Se}_{y}$ crystals synthesized by high pressure techniques [30]. It is worth mentioning that the pressure-stabilized cubic phase with $3: 4$ stoichiometry has also been theoretically predicted to undergo a SC transition with an estimated $T_{c} \simeq 3-4 \mathrm{~K}$ at $10 \mathrm{GPa}$ [26] (but not yet experimentally confirmed).

Only first-principles theories can be used to shed light on this complicated and rich physical scenario including multiple structural, electronic, SC, and topological phase transitions. We thus report a complete first-principles study of the structural, electronic, and SC properties of SnSe in a wide pressure range to understand its pressure phase diagram and the origin of the observed SC phase above $\simeq 27 \mathrm{GPa}$. To this aim, we address the conventional electron-phonon mediated superconductivity of the metallic phases (namely, the $\mathrm{Cmcm}$, the $\mathrm{CsCl}-$ type $P m \overline{3} m$, and the nonstoichiometric $\mathrm{Sn}_{3} \mathrm{Se}_{4} I \overline{4} 3 d$ phases), by means of highly accurate first-principles density-functional theory (DFT) of the normal state and its extension to the SC phase, the SuperConducting DFT (SCDFT) to predict the SC $T_{c}$. However, the careful comparison of computational predictions with available experiments revealed that the observed broad resistive transitions in Ref. [22] and the persistence of resistive tails complicates the theoretical interpretation.

Indeed, these effects are not uncommon in the recent literature on emergent SC materials and are typical features of systems where the SC state appears either at the interfaces between two insulators, as in $\mathrm{SrTiO}_{3}$-based interfaces [31-34], or in layered systems like transition-metal dichalcogenides [35-37] or twisted graphene [38]. A common feature for all these systems is the presence of a structural strain induced in the crystalline structure, due to the mismatch with the underlying substrate. Even though the microscopic mechanism responsible for superconductivity can be rather different in these families of superconductors, a systemic emergence of sizable rounding of the resistive transition has been reported, followed by a pronounced resistive tail that in some cases saturates to a finite value as $T \rightarrow 0$. The rounding of the transition cannot be ascribed $[39,40]$ to usual paraconductivity effects due to Gaussian SC fluctuations [41], since these are effective only in close proximity to the transition, while one often observes a suppression of the resistance at temperature as large as twice the critical one. These features can be instead well captured by assuming that the SC background gets intrinsically inhomogeneous on mesoscopic length scales, such that the resistive transition itself should be ascribed to a percolative mechanism of SC regions embedded into a non-SC matrix. Here we propose that a similar mechanism can be at play in SnSe. In addition, nonhydrostatic pressure conditions (possibly at work in Ref. [22]) are explored, reporting a sizable enhancement (nearly doubling) of $T_{c}$ due to strain field. This makes $\mathrm{SnSe}$ a peculiar system in which the $\mathrm{SC} T_{c}$ is strongly linked to structural properties.

The present paper is organized in the following structure: in Sec. II, we describe the computational techniques and in Sec. III we report our results, presenting the structural phase diagram under pressure (Secs. III A and III B), the electronic properties (Sec. III C), and SC ones (Sec. IIID). After the comparison with experimental critical temperatures, we list hypotheses to explain the observed disagreement and explicitly include inhomogeneities and nonhydrostatic effects (Secs. III E and IIIF). Section IV is devoted to conclusions and perspectives.

\section{COMPUTATIONAL DETAILS}

First-principles calculations have been performed within the DFT. We used ultrasoft pseudopotentials to describe the electron-ion interaction and plane-wave expansion of the Kohn-Sham wave functions as implemented in the QUANTUM ESPRESSO package [42,43]. The local density approximation (LDA) in the Perdew and Zunger parametrization has been adopted for the exchange-correlation potential [44]. The kinetic energy cutoff for plane-wave expansion has been set to 40 Ry (400 Ry for the charge density). Different MonkhorstPack [45] wave-vector grids were adopted, depending on the crystalline phase, ensuring a total energy convergence of $1 \mathrm{meV} /$ atom for all the considered phases. The MonkhorstPack mesh was set to $4 \times 10 \times 10$ for Pnma GeS-type, 
$4 \times 8 \times 6$ for $\gamma$-Pnma, $8 \times 8 \times 8$ for Cmcm, $10 \times 10 \times 10$ for $P m \overline{3} m$ CsCl-type, and $9 \times 9 \times 9$ for $F m \overline{3} m$ NaCl-type phases. The $I \overline{4} 3 d \mathrm{Sn}_{3} \mathrm{Se}_{4}$ phase has been simulated using a primitive cell with 14 atoms, and a Monkhorst-Pack mesh of $3 \times 3 \times 3$ was used for the self-consistent calculations.

The structural phase diagram was constructed calculating the enthalpy $H(P)=E[V(P)]+P V(P)$ as a function of the pressure. The calculations were performed fitting the ab initio total energy $E(V)$ as a function of the volume for each of the considered phases by means of a third-order BirchMurnaghan equation of state [46,47], and subsequently determining the corresponding $P(V)$ from its analytic derivative. Spin-orbit coupling (SOC) has not been taken into account in the structural phase diagram, while it has been included for the analysis of the electronic structure of the $P m \overline{3} m$ CsCl-type phase.

Phonon frequencies and electron-phonon coupling (EPC) matrix elements were determined within the densityfunctional perturbation theory [48]. A $3 \times 3 \times 3$ and $8 \times 8 \times$ 8 phonon wavevector grid were used for $C m c m$ and $P m \overline{3} m$ CsCl-type phases, respectively, while a $3 \times 3 \times 3$ phonon grid was used for the $I \overline{4} 3 d \mathrm{Sn}_{3} \mathrm{Se}_{4}$ phase. The Eliashberg function, $\alpha^{2} F(\omega)$, and the EPC parameter, $\lambda$, were evaluated as

$$
\begin{aligned}
\alpha^{2} F(\omega)= & \frac{1}{N\left(E_{F}\right)} \sum_{\mathbf{q} \nu} \sum_{m n} \delta\left(\omega-\omega_{\mathbf{q} \nu}\right) \\
& \times \sum_{\mathbf{k}}\left|g_{\mathbf{k}+\mathbf{q}, \mathbf{k}}^{\mathbf{q}, m n}\right|^{2} \delta\left(\epsilon_{\mathbf{k}+\mathbf{q}}^{m}-E_{F}\right) \delta\left(\epsilon_{\mathbf{k}}^{n}-E_{F}\right)
\end{aligned}
$$

and

$$
\lambda=2 \int \frac{\alpha^{2} F(\omega)}{\omega} d \omega
$$

through integration of the squared electron-phonon matrix element $\left|g_{\mathbf{k}+\mathbf{q}, \mathbf{k}}^{\mathbf{q} v, m n}\right|^{2}$ over a $40 \times 40 \times 40$ and $12 \times 12 \times 12$ electronic k-grid for the $\mathrm{CsCl}$-type and $\mathrm{Cmcm}$ phases, respectively, while $9 \times 9 \times 9$ grids were adopted through electronphonon interpolation in the $\mathrm{Sn}_{3} \mathrm{Se}_{4}$ phase. A gaussian approximation of the delta function with a smearing of $\sigma=0.015$ Ry was assumed for the evaluation of the EPC parameter $\lambda$.

The SC critical temperature mediated by electron-phonon interaction has then been calculated in the framework of SCDFT [49-54], in a fully ab initio way. The Coulomb interaction is accounted self-consistently from first-principles and on equal footing with respect to the electron-phonon interaction, in the static isotropic approximation. The isoenergy average of the screened Coulomb matrix elements $V_{\mathbf{k}, \mathbf{k}+\mathbf{q}}^{m, n}$, entering the gap equation is defined as

$$
V_{c}\left(\epsilon, \epsilon^{\prime}\right)=\frac{\sum_{\mathbf{k}, \mathbf{q}} \sum_{m, n} V_{\mathbf{k}+\mathbf{q}, \mathbf{k}}^{m, n} \delta\left(\epsilon_{\mathbf{k}+\mathbf{q}}^{m}-\epsilon\right) \delta\left(\epsilon_{\mathbf{k}}^{n}-\epsilon^{\prime}\right)}{\sum_{\mathbf{k}, \mathbf{q}} \sum_{m, n} \delta\left(\epsilon_{\mathbf{k}+\mathbf{q}}^{m}-\epsilon\right) \delta\left(\epsilon_{\mathbf{k}}^{n}-\epsilon^{\prime}\right)} .
$$

Screened Coulomb matrix elements were evaluated within random phase approximation as in Ref. [55]. The Coulomb pseudopotential $\mu$, giving an estimation of the strength of the Coulomb potential, is defined as the double average over the Fermi level,

$$
\mu=N\left(E_{F}\right) \ll V_{c}\left(\epsilon, \epsilon^{\prime}\right) \gg_{E_{F}},
$$
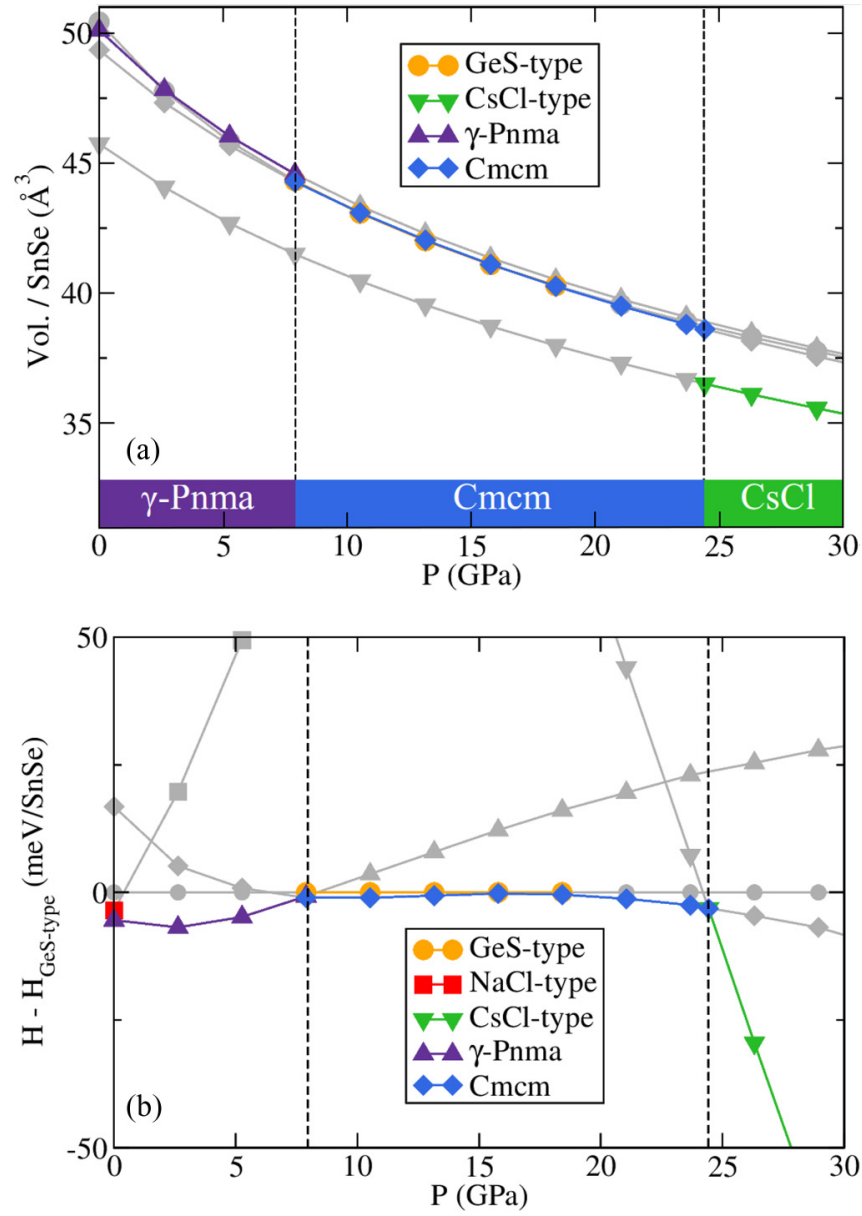

FIG. 2. Volume-pressure [panel (a)] and enthalpy-pressure phase diagram of SnSe [panel (b)]. Vertical dashed lines highlight $\gamma$-Pnma to $\mathrm{Cmcm}$ and $\mathrm{Cmcm}$ to $\mathrm{Pm} \overline{3} \mathrm{~m} \mathrm{CsCl}$-type structural transitions.

where $N(\epsilon)$ is the electronic density of states (DOS) and $E_{F}$ is the Fermi level.

\section{RESULTS AND DISCUSSION}

\section{A. Phase diagram}

In Fig. 2, we report the volume-pressure and enthalpypressure phase diagram of SnSe as obtained within the DFTLDA. According to experimental and previous computational studies $[5,21,22,24]$, we considered the following competing crystal structures: (i) cubic $F m \overline{3} m$ NaCl-type and $P m \overline{3} m$ CsCl-type, and (ii) orthorhombic Pnma GeS-type, $\gamma$-Pnma and $\mathrm{Cmcm}$ structures. The NaCl-type and the $\gamma$-Pnma structures are quasidegenerate at zero pressure, showing an enthalpy difference within $1 \mathrm{MeV} / \mathrm{SnSe}$, and both of them are slightly favoured with respect to the GeS-type phase (enthalpy difference being $\simeq 4 \mathrm{MeV} / \mathrm{SnSe}$ ). This result is in line with the calculations in Ref. [12], where the GeS-type and NaCl-type phases were found to be nearly degenerate $(0.78 \mathrm{MeV} / \mathrm{SnSe})$ within the generalized-gradient approximation and including SOC. The NaCl-type structure becomes rapidly unstable upon applying pressure, in favor of three competing structures within $10 \mathrm{MeV} / \mathrm{SnSe}$ up to $10 \mathrm{GPa}$ : the Pnma GeS-type, the $C m c m$, and the $\gamma$-Pnma phase. We find that the $\gamma$-Pnma 
phase is the ground state up to $8 \mathrm{GPa}$. This result is in line with the calculations of Ref. [31], in which $\gamma$-Pnma was predicted as the lowest energy structure in the range 1-8 GPa and with the XRD measurement interpreted with the above three phases' coexistence scenario. The latter then becomes unfavorable above $\simeq 10 \mathrm{GPa}$, while the orthorhombic Pnma GeS-type and $\mathrm{Cmcm}$ phases compete up to $20 \mathrm{GPa}$, the $\mathrm{Cmcm}$ phase being slightly favored above $\simeq 5.8 \mathrm{GPa}$. This last prediction is consistent with XRD measurements, whose spectra can be ascribed to $\mathrm{Cmcm}$ structures above $15.5 \mathrm{GPa}$ [22]. Looking at the electronic DOS of the system, we find a semiconductor-metal $\left[\mathrm{DOS}\left(E_{F}\right)=0.065\right.$ states $/ \mathrm{eV} / \mathrm{SnSe}$ at $6 \mathrm{GPa}$ transition in correspondence to the GeS-type to $\mathrm{Cmcm}$ symmetrization. This is in agreement with the experimental observation of a metallic behavior at low temperatures in the pressure range 5.4-9 GPa, reported in Ref. [22].

Finally, we predict a structural phase transition to the cubic $\operatorname{Pm} \overline{3} m$ CsCl-type phase at $\simeq 24.5 \mathrm{GPa}$, again in fairly good agreement with the experimental XRD spectra, where signatures of the cubic phase appear around $27 \mathrm{GPa}$, and with DFT-GGA calculations, predicting a critical pressure of $26 \mathrm{GPa}$ [22]. The slight underestimation of the transition pressure in our calculations is in line with the known overbinding tendency of LDA functionals. This last transition is of the first order, being accompanied by a volume discontinuity [see Fig. 2(a)]. It is worth mentioning that XRD patterns reported in Ref. [22] have been interpreted as a superposition of the cubic and orthorhombic $\mathrm{Cmcm}$ phases in the pressure range of 27.3-43.4 GPa. No indication of such coexistence of phases above $\simeq 25 \mathrm{GPa}$ can be seen in the enthalpy-pressure phase diagram Fig. 2(a), that has been obtained assuming hydrostatic pressure. A possible explanation of the coexistence can be ascribed to the high-pressure medium used in XRD measurements in Ref. [22] (Daphne 7373), which becomes solid at room temperature after $\simeq 2 \mathrm{GPa}[56,57]$, possibly inducing nonhydrostatic pressure transmission effects [58]. The latter may, in turn, be responsible for structural mesoscopic inhomogeneities and, possibly, coexisting structural phases with different local pressures (see below).

\section{B. Pressure evolution of the lattice parameters}

A comparison between the experimental and the theoretical lattice parameters as a function of the pressure is reported in Fig. 3. The first-principles results are in excellent quantitative agreement with the experiments [21,22], the difference being of the order of $1 \%$. We also report the evolution of the internal coordinates of the GeS-type phase, $z(\mathrm{Sn})$ [Fig. 3(b)], which can be considered as the order parameter of the structural transition from the GeS-type to the $\mathrm{Cmcm}$ phase [21]: The experimental trend is well reproduced even in this case. The GeS-type phase gradually symmetrizes under pressure both in the ab initio calculations and in the experimental data by Loa et al. [21], in which the GeS-type phase disappears after $10.5 \mathrm{GPa}$. Other studies, though, report a coexistence between the two structures up to $\simeq 13.4 \mathrm{GPa}$ [24]. Interestingly, and at variance with Ref. [22], in the experiments of Zhou et al., the transmitting medium is a gas (Neon) [24], which should provide higher degree of hydrostaticity. Thus the phase coexistence between GeS-type and $\mathrm{Cmcm}$ could be intrinsic
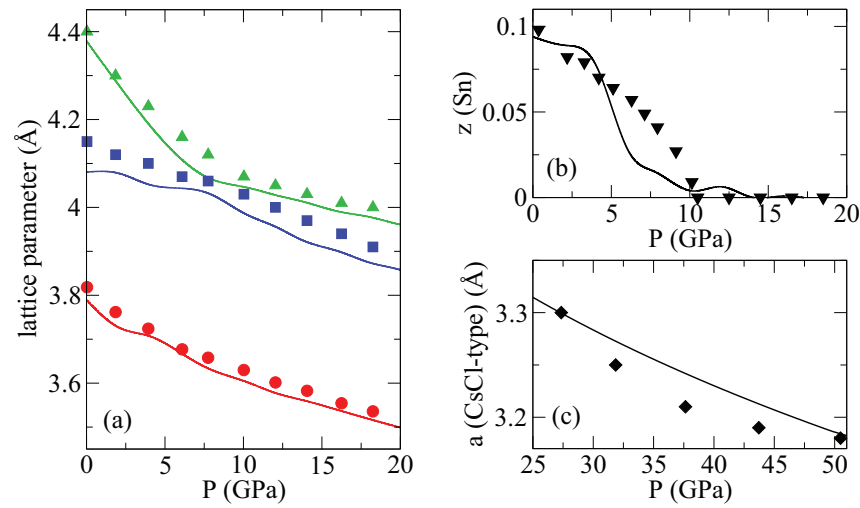

FIG. 3. Comparison between experimental (points) and theoretical (lines) pressure evolution of the lattice parameters of the GeS-type phase [panel (a)], experimental data from Ref. [22], the $z(\mathrm{Sn})$ coordinate of the GeS-type phase [panel (b)], and the lattice parameter of the CsCl-type phase [panel (c)], experimental data from Ref. [21].

(not due to nonhydrostatic effects) in line with theoretical results.

After $25 \mathrm{GPa}$, the predicted ground state is the $\mathrm{CsCl}-$ type cubic phase. The evolution of its lattice parameter with pressure is compared with the experiments of Ref. [22] and reported in Fig. 3(c) up to $50 \mathrm{GPa}$, again with an excellent agreement.

The successful prediction of the stability of the CsCl-type phase and of the experimental lattice parameters support the reliability of our theoretical analysis, thus motivating the study of this system at high pressure, in particular its SC phase.

\section{Electronic properties of the CsCl-type phase}

First we study the electronic band structure of the $\mathrm{CsCl}$ type phase. The nonrelativistic electronic band structure at $50 \mathrm{GPa}$ is reported in Fig. 4(a). A Dirac line node (DLN) is found around the high-symmetry point $\mathrm{X}$ in the M-X-R plane of the Brillouin zone, arising from the crossing of $\mathrm{Sn}$ $s$ - and $p$-orbital bands. The DLN is located between 0.85 and $1 \mathrm{eV}$ above the Fermi level in the relevant pressure range (30-50 GPa). As expected, SOC allows for hybridization between the two bands, causing a splitting of the DLN. As argued in Ref. [22], the gap at DLN is topologically nontrivial, belonging to the $Z_{2}$ topological class, as highlighted by the band inversion that can be seen from the atomic projections on the Sn $s$ - and p-orbitals displayed in Figs. 4(b) and 4(c). We further report a Lifshitz transition with increasing pressure in correspondence of the $\mathrm{X}$ point, occurring at $\simeq 25 \mathrm{GPa}$, i.e., around the critical pressure for the structural transition from the $\mathrm{Cmcm}$ phase to the CsCl-type phase. As shown in Fig. 4(c), the X-centred electron Fermi surface derived from Sn $p$-orbitals disappears at higher pressure.

The evolution of the DOS with pressure for the CsCltype phase is reported in Fig. 5. At ambient pressure, the Fermi level is located in proximity of a Van Hove singularity, which corresponds to the band extremum visible in the $\Gamma-\mathrm{M}$ direction in Fig. 4(a). Its evolution with pressure is 

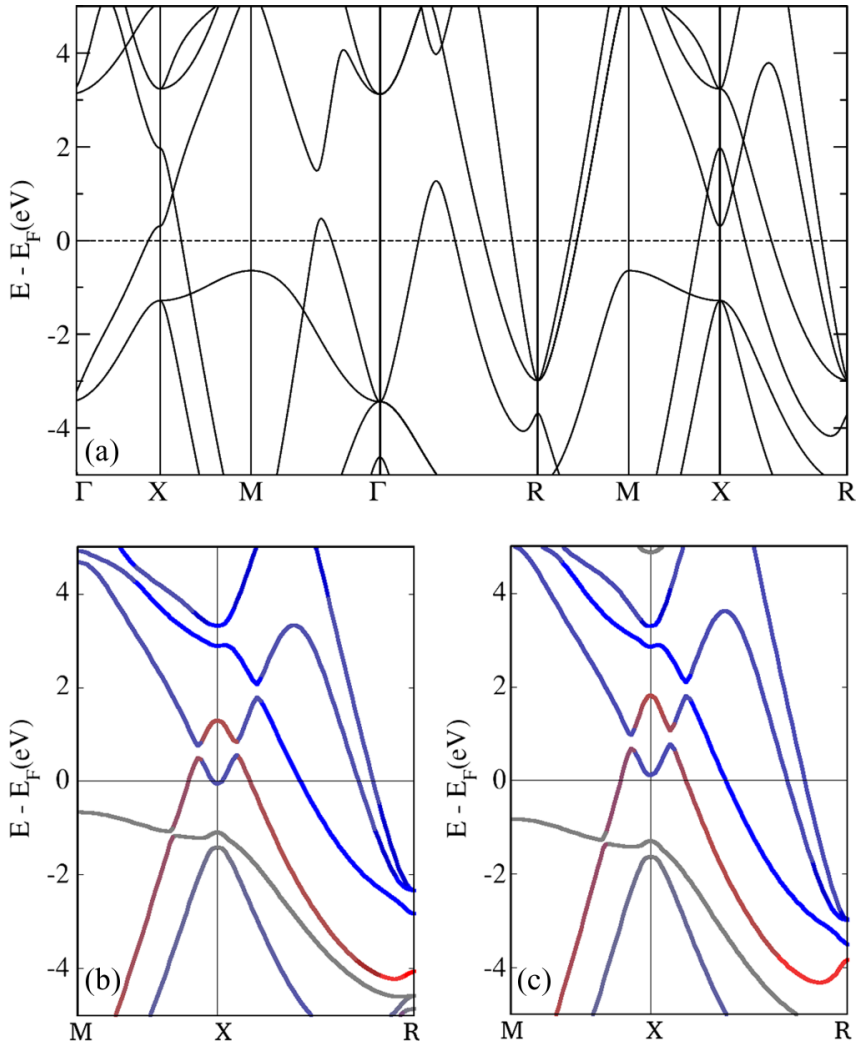

FIG. 4. Band structure of the CsCl-type phase of SnSe at $50 \mathrm{GPa}$ without SOC [panel (a)]. Band structure with SOC projected on atomic $\operatorname{Sn}(s)$ (red) and atomic $\operatorname{Sn}(p)$ (blue) orbitals at $20 \mathrm{GPa}$ [panel (b)] and $40 \mathrm{GPa}$ [panel (c)].

characterized by a widening of the bandwidth. The occupied states move toward lower energy, while the unoccupied states (in particular the Van Hove singularity) toward higher energies with a sensible broadening. The DOS at the Fermi level is found to decrease significantly as the pressure is increased, as shown in the inset of Fig. 5.

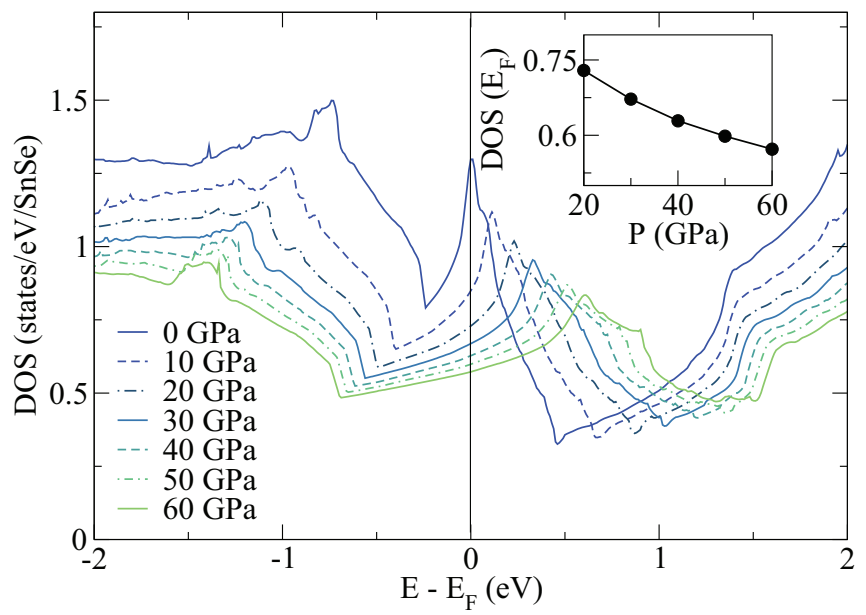

FIG. 5. Evolution of the DOS for the CsCl-type phase calculated at different pressures. Inset: DOS at Fermi level as a function of the pressure (states $/ \mathrm{eV} / \mathrm{SnSe}$ ).

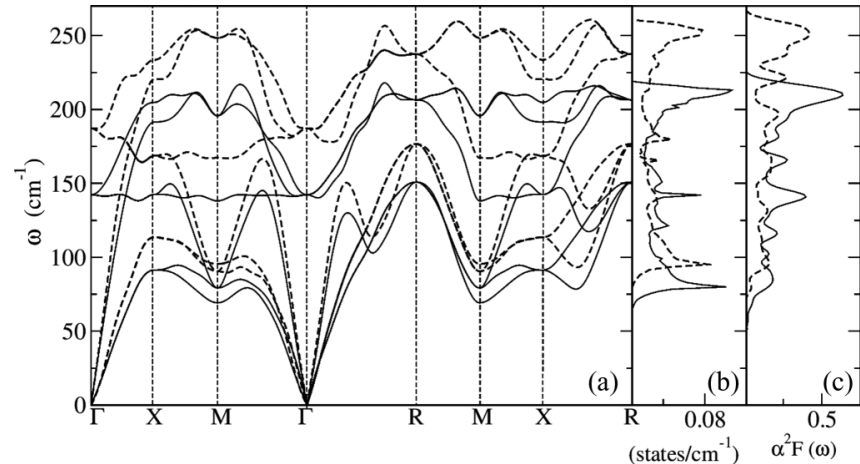

FIG. 6. Phonon dispersion for the CsCl-type phase at $30 \mathrm{GPa}$ (solid) and $50 \mathrm{GPa}$ (dashed) (panel (a)]. Corresponding DOS [panel (b)] and Eliashberg function [panel (c)].

\section{Superconducting transition}

Superconductivity was observed in SnSe at $3 \mathrm{~K}$ at $27 \mathrm{GPa}$. The critical temperature shows a domelike behavior as a function of the pressure. However, it varies only of $\simeq 0.5 \mathrm{~K}$, up to $50 \mathrm{GPa}$, resulting rather constant. This peculiar behavior is quite uncommon, in particular in view of the wide pressure range $[22,27,29]$ and considering the strong variation of the electronic properties (Fig. 4). To understand the origin of the SC transition and its nontrivial evolution as a function of the pressure, as well as to give hints on the conventional or unconventional nature of the SC phase, we calculated the phonon frequencies and the $\mathrm{EPC}$, in the CsCl-type phase between 10 and $60 \mathrm{GPa}$. As a first result, we present the phonon band structure at 30 and $50 \mathrm{GPa}$, Fig. 6. The CsCl-type phase is always dynamically stable, showing no imaginary phonon frequencies, thus further supporting its experimental attribution. Optical and acoustic branches strongly overlap due to a very dispersive acoustic mode and because of the similarity between Sn and Se atomic masses (118.71 a.u. and 78.96 a.u., respectively). The comparison between the phonon DOS and the Eliashberg function $\alpha^{2} F(\omega)$ [Eq. (1)] shown in Fig. 6(c) reveals that the EPC is distributed in all the frequency ranges, with the main peaks in $\alpha^{2} F(\omega)$ corresponding to DOS peaks.

The calculated EPC parameter is $\lambda=0.49$ at $30 \mathrm{GPa}$ and decreases with increasing pressure [see Table I and inset in

TABLE I. The electron-phonon coupling parameter $(\lambda)$, average Coulomb potential $(\mu)$, logarithmic average phonon frequency $\left(\omega_{\log }\right)$, electronic DOS at Fermi level $\left(\mathrm{DOS}_{E_{F}}\right)$, BCS ratio between the value of the energy gap at zero temperature and the value of the superconducting transition temperature $\left(\frac{2 \Delta_{\mathrm{SC}}(0)}{T_{C}}\right)$ for selected values of the pressure.

\begin{tabular}{lccccc}
\hline \hline$P(\mathrm{GPa})$ & $\lambda$ & $\mu$ & $\omega_{\log }(K)$ & $\operatorname{DOS}_{E_{F}}\left(\frac{\mathrm{eV}^{-1}}{\mathrm{spin}}\right)$ & $\frac{2 \Delta_{\mathrm{SC}}(0)}{T_{C}}$ \\
\hline 10 & 1.02 & 0.24 & 122.2 & 0.42 & 3.92 \\
20 & 0.64 & 0.21 & 168.4 & 0.36 & 3.54 \\
30 & 0.49 & 0.26 & 193.6 & 0.33 & 3.33 \\
40 & 0.40 & 0.19 & 216.1 & 0.31 & 3.1 \\
50 & 0.34 & 0.18 & 234.6 & 0.30 & 2.99 \\
60 & 0.30 & 0.18 & 253.1 & 0.29 & 2.9 \\
\hline \hline
\end{tabular}



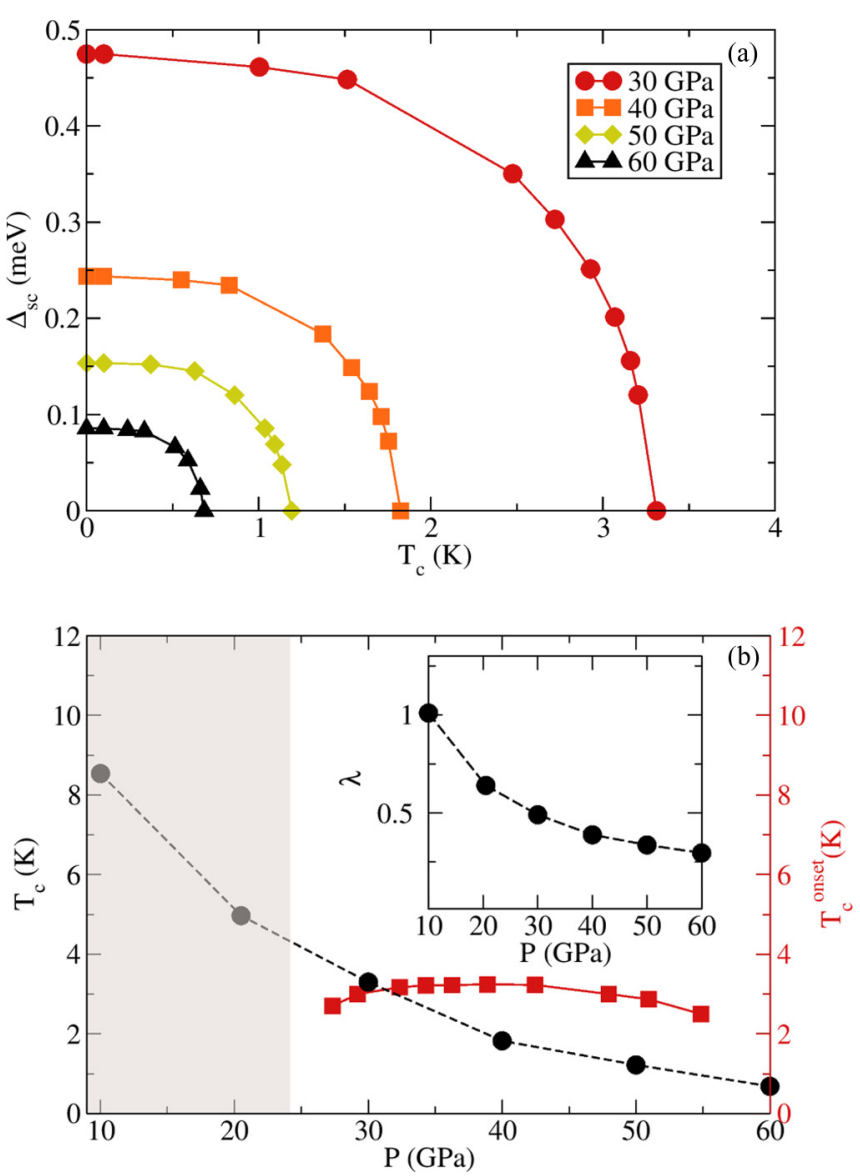

FIG. 7. Supeconducting gap $\left(\Delta_{\mathrm{SC}}\right)$ as a function of the temperature at various pressures [panel (a)]. Critical temperature of the $\mathrm{CsCl}$ type phase and $\lambda$ (inset) as a function of the pressure [panel (b)]. Red squares are the experimental values of the onset temperature. The shadowed area corresponds to the pressure range where the $\mathrm{CsCl}$ phase is not the ground state. Lines are a guide to the eye.

Fig. 7(b)]. The solution of the isotropic SCDFT gap equation [50,51], including the electron-phonon pairing $\alpha^{2} F(\omega)$ [Eq. (1)] and Coulomb repulsion [Eq. (3)], predicts $T_{c}=3.3 \mathrm{~K}$ at $30 \mathrm{GPa}$. This value is in very good agreement with the results reported Ref. [22] for the onset temperature $\left(T_{c}^{\text {onset }}\right)$ at which the resistance begins to decrease toward the zeroresistance limit. In Fig. 7(b), we report the SC gap as a function of the temperature $\Delta_{\mathrm{SC}}(T)$ for different pressures. The curve is BCS-like with a $\frac{2 \Delta_{\mathrm{SC}}(0)}{T}$ ratio always close to the BCS value (3.53) (Table I). However, we predict a detrimental pressure effect on SC properties (Fig. 7), which is in contrast with the experimental observation of an almost constant $T_{c}$ of $\simeq 3 \mathrm{~K}$ up to $50-55 \mathrm{GPa}$ [22]

We extended the calculation in the lower pressure region, where the $\mathrm{CsCl}$-type phase remains dynamically stable (metastable), finding that the critical temperature can be increased up to $\sim 8.5 \mathrm{~K}$ at $10 \mathrm{GPa}$ [Fig. 7(b)]. We ascribe the origin of the theoretical behavior of $T_{c}(P)$ to two distinct effects: the increasing of the characteristic phonon frequencies upon pressure, clearly visible in Fig. 6(c) and in Table I, and the decreasing of the electronic DOS at the Fermi level due to the widening of the band structure with pressure, as shown in the inset of Fig. 5. Both effects contribute to the significant suppression of $\lambda$ with increasing pressure.

We note that a similar trend has been calculated for the CsCl-type phase of SnTe [59] under pressure. The detrimental repulsive electron-electron Coulomb interaction, which can be estimated with the double average of the Coulomb-matrix element on the Fermi surface, on the contrary lowers with the pressure, mainly due to the decrease of $\operatorname{DOS}\left(E_{F}\right)$ (see Table I).

Although the predicted $T_{c}$ 's are in quantitative agreement with the measured ones, especially considering the complication of the experiment and the relatively low critical temperature, we wanted to further investigate the origin of the qualitative differences of $T_{c}(P)$ between theory and experiments, considering that the predictive power of SCDFT is much better for metals under pressure [51,53,54,60,61].

To this aim, we analyzed the consequences of some effects not included in our calculations: (i) spin-orbit coupling, (ii) multiband effects, and (iii) formation of metastable phases at high pressure.

(i) Spin-orbit coupling. Sn has a relatively high spin-orbit effect, which is ultimately responsible for the topological phase in CsCl-type structure. SOC was not considered in previous calculations, and to evaluate the effect of its inclusion, we calculate the phonon frequencies and $\lambda$ at $50 \mathrm{GPa}$, where the difference with experimental $T_{c}$ is larger. We do not observe any significant difference in $T_{c}$ with or without SOC. This fits a rationale once we note that electron bands at Fermi level, contributing to $\lambda$ through Eq. (2), are not affected much with the inclusion of the SOC, the most significant differences occurring at the Dirac line node $\left(\simeq 1 \mathrm{eV}\right.$ above $\left.E_{F}\right)$. Moreover, the phonon spectrum shows no relevant differences with and without the inclusion of SOC (not shown).

(ii) Multiband superconductivity. We considered the possibility of a sizable multiband superconductivity effect in the CsCl-type phase, which can be envisaged due to its disconnected Fermi surface (Fig. 8). To this aim, we assumed a two-band model, including the folded band centered around the $\Gamma$ point of the Brillouin zone in the first group (blue Fermi surface in Fig. 8) and the three sheets around the R point in the second group (red Fermi surfaces in Fig. 8). In a multiband superconductor, the coupling parameter is defined by the $\lambda$

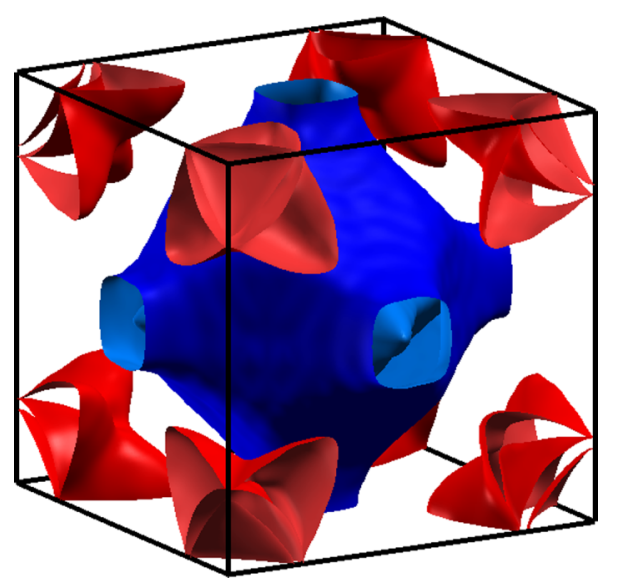

FIG. 8. Fermi surface for the CsCl-type phase at $30 \mathrm{GPa}$. $\Gamma$ and $\mathrm{R}$ points are at the center and at the corner of the cube, respectively. 
matrix whose matrix elements are

$$
\lambda_{i, j}=2 \int_{0}^{\infty} d \omega \frac{\alpha^{2} F(\omega)_{i, j}}{\omega},
$$

with $i, j$ band indexes and $\alpha^{2} F(\omega)_{i, j}$ the $(i, j)$ resolved Eliashberg function [62] normalized by the factor $\frac{N\left(E_{F}\right)}{N_{i}\left(E_{F}\right)}$, where $N_{i}\left(E_{F}\right)$ is the partial DOS at $E_{F}$ due to the $i$ th band. From the calculation of the highest eigenvalue of $\lambda$ matrix (which determines the critical temperature), we found that the multiband solution doesn't differ much with respect to the single band approximation ( $\simeq 0.01$ variation in $\lambda$ value $)$, ruling out this hypothesis.

(iii) Superconductivity in competing structural phases. In view of the complex pressure phase diagram of $\mathrm{SnSe}$ and the numerous phase transitions observed, formation of metastable phases cannot be excluded. Indeed, notable examples of superconductivity in metastable phases were recently found in Refs. [54,63,64].

The first competing phase we considered is the orthorhombic $\mathrm{Cmcm}$, observed to coexist (at room temperature) with CsCl-type phase up to $\simeq 43.4 \mathrm{GPa}[22]$, in the range of pressures where the superconductivity is observed. To verify this hypothesis, we checked the dynamical stability of the $\mathrm{Cmcm}$ phase at high pressure, calculating the phonon dispersions at 40 and $50 \mathrm{GPa}$ (not shown). We confirm its metastability (dynamically stable) and the possibility of coexistence between the two phases after the enthalpy transition. However, $\lambda$ is rather small $(\lambda \simeq 0.25$ and $\lambda \simeq 0.28$ at 40 and $50 \mathrm{GPa}$, respectively), eventually leading to vanishing values of $T_{c}$. Interestingly, $\lambda$ increases with pressure at variance with what observed in CsCl-type phase. These findings are compatible with the absence of SC transition between $\simeq 12-25 \mathrm{GPa}$ [22], where metallic $\mathrm{SnSe}$ adopts the $\mathrm{Cmcm}$ structure, at the same time excluding any additional contribution to superconductivity arising from the possibly coexisting $\mathrm{Cmcm}$ phase in the higher-pressure range.

The second metastable phase we considered is the recently predicted $\mathrm{Sn}_{3} \mathrm{Se}_{4}$ compound [26,65]. We focused on the $\mathrm{Sn}_{3} \mathrm{Se}_{4}$ structure with cubic $I \overline{4} 3 d$ symmetry, that has been proposed to be stable between 18-70 GPa [26]. Based on the convex hull reported in Refs. [26,65], we assumed that SnSe could spontaneously change stoichiometry at a certain pressure, although XRD does not show any evidence of this process. Nonetheless, the predicted SC critical temperature at $40 \mathrm{GPa}$ is $0.86 \mathrm{~K}(\lambda=0.41)$, still too low to justify the experimental observations.

\section{E. The effective medium approximation for the random-resistor network}

Although we explored various hypotheses as the origin of the observed discrepancy in the $T_{c}(P)$ curve between experiments and theory, our theoretical results seem solid within the conventional electron-phonon mechanism. At this point, we will consider possible experimental (spurious) effects not included in the theoretical models. A possible explanation for the discrepancies between predicted and measured $T_{c}(P)$ could be ascribed to the presence of mesoscopic inhomogeneities caused by nonhydrostatic pressure conditions in the experimental setup, due to the solid transmitting medium
$(\mathrm{NaCl})$ used in Refs. [22,29]. This effect has been recently documented in Ref. [66]. As suggested in Sec. III A, such nonhydrostatic effects might explain the coexistence of (non$\mathrm{SC}$ ) $\mathrm{Cmcm}$ and (SC) CsCl-type phases emerging from XRD spectra and can induce strain fields which can, in principle, determine different SC phases [67]. Indeed, signatures of such inhomogeneous character of the system in the pressure range 27.3-43.4 GPa could be envisaged in the measured resistance drops, which appear significantly broadened for $P \lesssim 43.4 \mathrm{GPa}$ and display pronounced tails compatible with percolative superconductivity $[39,68]$, that may develop in systems displaying coexisting metallic and $\mathrm{SC}$ regions. We will investigate this effect in this section by means of an effective model.

Following the same approach used for SC interfaces [39,68,69] or SC transition-metal dichalcogenides [40], we describe here the inhomogeneous SC state of high-pressure $\mathrm{SnSe}$ as a random resistor network of resistances $R_{i}$ on a regular $d$-dimensional lattice. In this picture, every resistor $R_{i}$ of the network represents a mesoscopic region of the system, whose local critical temperature $T_{c}^{i}$ is randomly distributed. The global resistance $R_{\mathrm{em}}$ of the system is given, within the effective medium approximation (EMA), as a solution of the following self-consistent equation in $d$ dimensions [70]:

$$
\sum_{i} \frac{R_{\mathrm{em}}-R_{i}}{R_{\mathrm{em}}+(d-1) R_{i}}=0 .
$$

Here we assume that each resistor can take only two constant values: $R_{i}=R_{N}$ if the link is in the normal state and $R_{i}=0$ if the temperature is lowered below the bond critical temperature $T_{c}^{i}$, so the temperature dependence in each bond will be $R_{i}=$ $R_{N} \theta\left(T-T_{c}^{i}\right)$, where $\theta(x)$ is the Heavyside step function. If we denote by $G\left(T_{c}^{i}\right)$ the probability distribution of the local critical temperatures, the overall probability $w(T)$ that the local resistance $R_{i}=0$ is given by

$$
w(T)=\int_{T}^{+\infty} G\left(T_{c}^{i}\right) d T_{c}^{i} .
$$

Equation (6) can then be solved explicitly at each temperature,

$$
R(T)=R_{N}[1-(d-1) w(T)],
$$

showing that the transition to the SC state occurs when $w\left(T_{c}\right)=1 /(d-1)$. As has been widely discussed in Refs. [39,40,68,69] in the presence of a distribution of local $T_{c}^{i}$ the resistance [Eq. (8)] starts to progressively deviate from the normal-state value $R_{N}$ as a larger fraction $w(T)$ of the system becomes $\mathrm{SC}$. When the total $\mathrm{SC}$ fraction, i.e., $w_{s}=$ $\int G\left(T_{c}^{i}\right) d T_{c}^{i}$, is equal to one, so all the system is potentially $\mathrm{SC}$, the transition occurs in $d$ dimensions when $1 /(d-1)$ bonds switch to the SC state. However, whenever a fraction of the system stays metallic at all temperatures, i.e., $w_{s}<$ 1, a larger part of the bonds have to turn SC to reach the percolation threshold. In this situation, as $T$ decreases, $R(T)$ shows first a significant drop and then a pronounced tail that can even saturate to a finite $R(T \rightarrow 0)$ value when $w_{s}<$ $1 /(d-1)$. Within the EMA, the existence of resistive tails is then indicative of the existence of a large non-SC fraction of the system. 


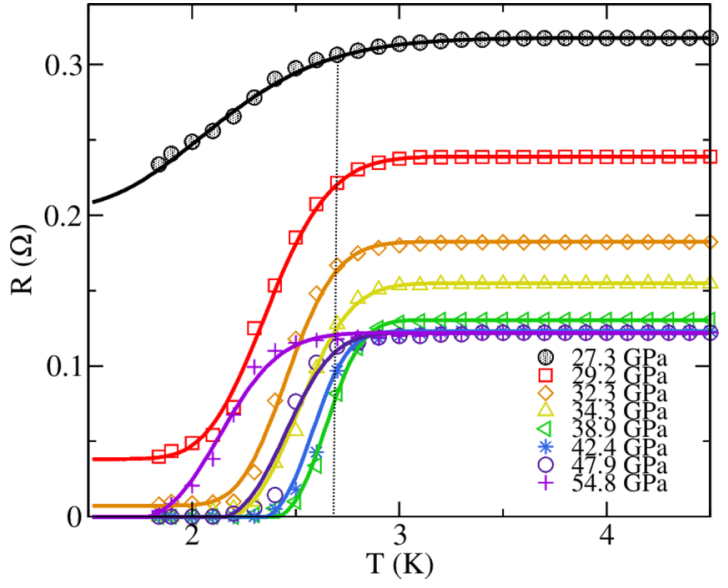

FIG. 9. EMA fit of the experimental resistance data from Ref. [22] at different values of the nominal pressure. The dashed line indicates a representative value of $T_{c}^{\text {onset }}$ at $27.3 \mathrm{GPa}$.

Here we assume that the $T_{c}^{i}$ inhomogeneity arises from an inhomogeneous distribution of the local pressure $P$. For the sake of concreteness, we will consider a Gaussian distribution $g(P)$ for the local pressure:

$$
g(P)=\frac{1}{\sqrt{2 \pi} \sigma} e^{-\frac{\left(P-P_{m}\right)^{2}}{2 \sigma^{2}}},
$$

where $P_{m}$ denotes the average pressure and $\sigma$ its variance. We used our calculated $a b$ initio critical temperatures as the local values of $T_{c}$ at different pressures, which can be well fitted as

$$
T_{c}=a e^{-b P},
$$

where $a=14.0 \mathrm{~K}$ and $b=0.050 \mathrm{GPa}^{-1}$. It then easily follows that the probability distribution $G\left(T_{c}^{i}\right)$ appearing in Eq. (7) is $G\left(T_{c}^{i}\right)=g\left(P\left(T_{c}^{i}\right)\right) / b T_{c}^{i}$. Finally, to account for the existence of pronounced resistive tails, we will normalize the total $G\left(T_{c}^{i}\right)$ to a parameter $w_{s}$ that can be smaller than 1 . We then have, for each nominal external pressure, three fitting parameters: $P_{m}, \sigma$, and $w_{s}$.

The theoretical fit of the experimental resistance data extracted from Ref. [22] based on the EMA for $d=3$ is shown in Fig. 9. The model accounts very well for all the main features observed experimentally at different external pressure. As shown in Fig. 10, where we report the fitted parameters, the SC fraction of the system $w_{s}$ (shown in the inset) remains always relatively small, and for $P<34 \mathrm{GPa}$ is even smaller than the percolation threshold, 0.33 , in three dimensions. As a consequence, the resistance displays a sizable drop but then saturates to a finite value, since a percolative $\mathrm{SC}$ pattern cannot be established in the system. However, the most remarkable result is the apparent discrepancy between the nominal external pressure and the local pressure of the $\mathrm{SC}$ regions, which fluctuate around the $P_{m}$ value. As shown in Fig. 10, the first SC signature appears with a local pressure $P_{m}$ rather larger than the nominal one, with large relative fluctuations $\sigma$ and a very small SC fraction $w_{s}$. It then appears that the system tries to avoid the coexistence region between the two phases found in $a b$ initio calculations. The way out to the metastable phase seems to be the tendency to enucleate few regions where the local $P$ is large enough to stabilize the

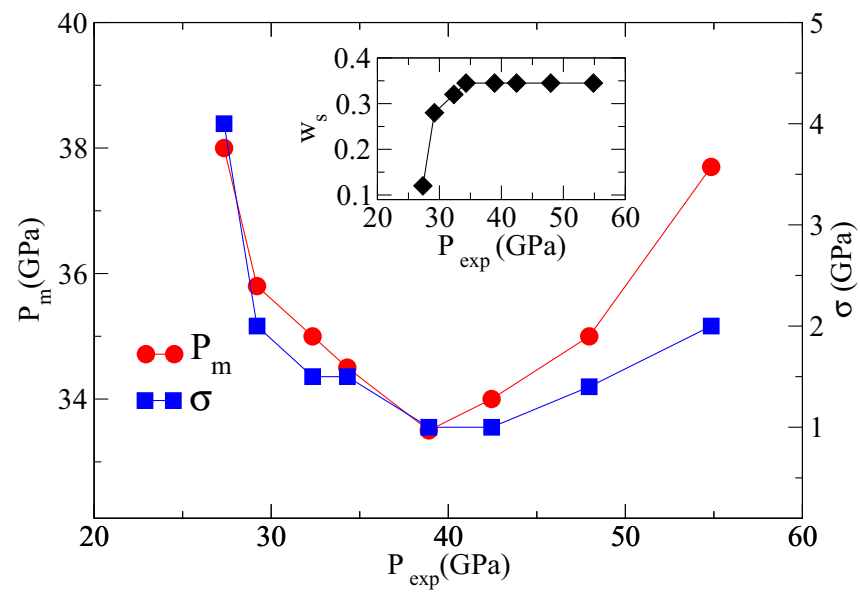

FIG. 10. Parameters $\left(P_{m}, \sigma\right)$ extracted from the fit of resistance curves shown in Fig. 9 with Eq. (8). In the inset, the SC fraction $w_{s}$.

SC phase. As the external pressure increases, the system tends to be more homogeneous, with a decrease of $\sigma$, an increase of the SC fraction and a decrease of the local $P$ in the SC region. Once the percolation threshold has been reached, the SC fraction stays constant and the local pressure in the SC regions starts to monotonically increase following the increase of the external nominal pressure. The above results demonstrate that predicted critical temperatures as a function of the pressure, $T_{c}(\mathrm{P})$, are indeed compatible with the experiments, once the effect of inhomogeneities is taken into account.

\section{F. The effect of nonhydrostatic conditions}

However, apart from pressure and phase inhomogeneities, the nonhydrostatic pressure conditions (due to transmitting medium and phase coexistence) can in principle change the SC critical temperatures, adding ingredients to explain the form of resistance curves as a function of the pressure and of the temperature, and giving a definite degree of indetermination for the mean pressure. We further explored this interesting scenario to understand if and how nonhydrostatic conditions modify the SC critical temperature with respect to the previous prediction under hydrostatic conditions.

To verify this hypothesis, we consider the CsCl-type phase at $50 \mathrm{GPa}$, simulating realistic nonhydrostatic conditions inducing a rhombohedral deformation of the cubic CsCl-type structure, which, varying the angle between the lattice vectors of the unit cell is in general energetically favored [71]. Considering the hydrostatic nominal pressure of $50 \mathrm{GPa}$, for each rhombohedral angle, we calculated the stress tensor and optimized the lattice parameters to achieve a mean pressure (trace of the stress tensor) of $50 \mathrm{GPa}$.

For selected nonhydrostatic conditions, we calculated the dynamical properties, the EPC, and the SC critical temperature within the same theoretical and computational scheme used in Sec. IIID (see Fig. 11). Indeed, we find that the critical temperature strongly depends on the nonhydrostatic conditions: Increasing and decreasing the rhombohedral angle has a positive effect on the critical temperature, which nearly doubles for $\theta \simeq 84^{\circ}$. This result is not so common for SC materials, where the structural deformations are in general 


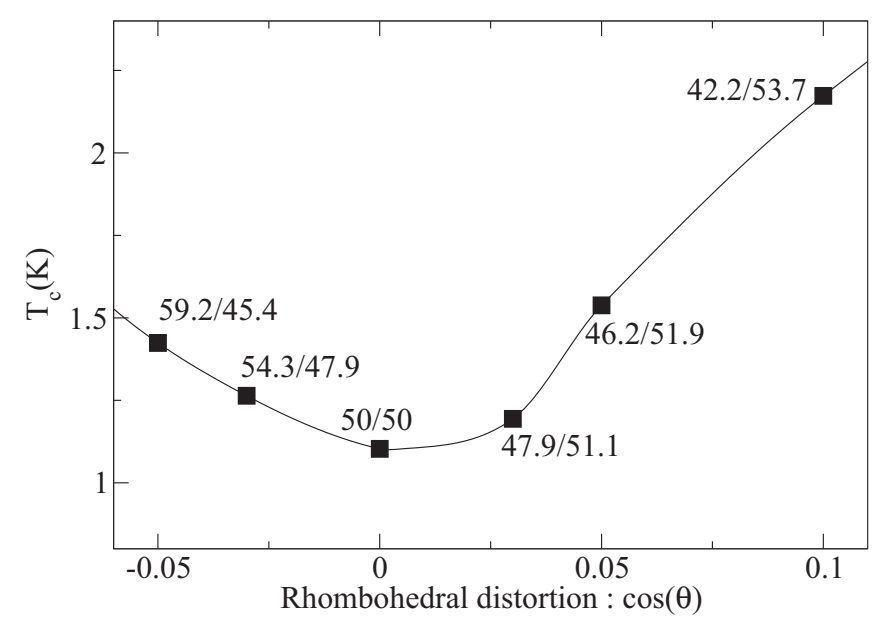

FIG. 11. Superconducting critical temperature as a function of the rhombohedral angle at $50 \mathrm{GPa}$. Numbers near the symbols represent the in-plane/out-plane components of the stress tensor in the rhombohedral phase in the hexagonal representation.

detrimental for the SC phase and represents a peculiar effect at work in SnSe. By the way, the predicted $T_{c}$ at $50 \mathrm{GPa}$ under this conditions is $\simeq 2.5 \mathrm{~K}$, which is now in line with the experiments. Although the considered deformation may represent a model system, the calculation highlights the positive effect that nonhydrostatic pressure conditions have on $T_{c}$, which can be eventually exploited with dedicated experiments.

\section{CONCLUSIONS}

The study of the structural properties of SnSe led us to unexpected and interesting findings. First of all, the phase diagram shows a unique situation in which different structures coexist in a small enthalpy range up to $15 \mathrm{GPa}$. We find that GeS-type phase symmetrizes into the $\mathrm{Cmcm}$ phase at $\simeq 6 \mathrm{GPa}$ by a second-order phase transition accompanied by a semiconductor-metal transition, in agreement with the experimental result. A first-order structural transition takes place at $24.5 \mathrm{GPa}$, as recently experimentally reported [22], from the $\mathrm{Cmcm}$ to the CsCl-type structure accompanied by an electronic Lifshitz transition. This high-pressure phase is SC and topologically nontrivial with a calculated $T_{c}$ of the same order of the one experimentally observed. We predict a suppression of $T_{c}$ as a function of the pressure, at variance with the experimental results [22] which show a "nearly" constant $T_{c}$ from 27 to $50 \mathrm{GPa}$. However, the rather low values of $T_{c}$ and the substantial differences between the onset and zero resistance $T_{c}$ [22,29], give rise to considerable relative uncertainties. Indeed, we found that inclusion of mesoscopic inhomogeneities due to the coexistence of the $\mathrm{Cmcm}$ phase and the CsCl-type phase accounts for the experimentally observed broadening of the resistance curves, interpreted by means of a percolative random-resistor network model, analyzed within the effective medium model. The model fits the experimental resistance curves with a set of parameters perfectly in line with experimental conditions, indicating that it probably grasps the most important physical effects. We further explored the effect of nonhydrostatic pressure conditions on the SC critical temperature, finding that rhombohedral deformations at $50 \mathrm{GPa}$ have sizable (positive) effect on $T_{c}$, enhancing it close to the observed one. We mention that, in the process of justifying critical temperatures observed in $\mathrm{SnSe}$, we found that the $\mathrm{Sn}_{3} \mathrm{Se}_{4}$ compound is $\mathrm{SC}$ at $40 \mathrm{GPa}$ with a critical temperature of $0.86 \mathrm{~K}$, in a pressure range where the compound is stable in the convex hull. Based on present theoretical and experimental evidences, we conclude proposing two possible experimental studies to clarify the origin of superconductivity in SnSe. To understand the possible role of metastable phases, the SC phase transition can be measured, lowering the temperature from high toward low $(\simeq 1 \mathrm{~K})$ temperature for each pressure. This will ensure that the lowest enthalpy phase will be reached at each pressure. In addition, increasing the pressure at low temperature and measuring the SC $T_{c}$, increasing the temperature up to the transition temperature [72], the metastable phases can be promoted. In a second experiment, we propose to explore the existence of pressure hysteresis following the SC phase diagram starting from high pressure $(P=50 \mathrm{GPa})$ and releasing the pressure at low $T$. This could allow the stabilization of the CsCl-type phase at lower pressures where it is metastable and in which we predicted an higher $T_{c}(8.5 \mathrm{~K})$ than what was observed in Ref. [22] at higher pressures.

\section{ACKNOWLEDGMENTS}

We acknowledge financial support from the Italian Ministry for Research and Education through PRIN-2017 project "Tuning and understanding Quantum phases in 2D materials - Quantum 2D” (IT-MIUR Grant No. 2017Z8TS5B) and CINECA (ISCRA initiative) for computing resources. C.T. acknowledges the French state funds managed by the ANR within the Investissements d'Avenir program under Reference No. ANR-11-IDEX-0004-02, and more specifically within the framework of the Cluster of Excellence MATISSE led by Sorbonne Université. L.B. acknowledges the Italian MAECI under the Italian-India collaborative Project No. SUPERTOPPGR04879.
[1] L.-D. Zhao, S.-H. Lo, Y. Zhang, H. Sun, G. Tan, C. Uher, C. Wolverton, V. P. Dravid, and M. G. Kanatzidis, Nature 508, 373 (2014).

[2] L.-D. Zhao, G. Tan, S. Hao, J. He, Y. Pei, H. Chi, H. Wang, S. Gong, H. Xu, V. P. Dravid, C. Uher, G. J. Snyder, C. Wolverton, and M. G. Kanatzidis, Science 351, 141 (2015).
[3] D. Ibrahim, J.-B. Vaney, S. Sassi, C. Candolfi, V. Ohorodniichuk, P. Levinsky, C. Semprimoschnig, A. Dauscher, and B. Lenoir, Appl. Phys. Lett. 110, 032103 (2017).

[4] U. Aseginolaza, R. Bianco, L. Monacelli, L. Paulatto, M. Calandra, F. Mauri, A. Bergara, and I. Errea, Phys. Rev. Lett. 122, 075901 (2019). 
[5] J. Yan, F. Ke, C. Liu, L. Wang, Q. Wang, J. Zhang, G. Li, Y. Han, Y. Ma, and C. Gao, Phys. Chem. Chem. Phys. 18, 5012 (2016).

[6] L. C. Gomes and A. Carvalho, Phys. Rev. B 92, 085406 (2015).

[7] A. S. Rodin, L. C. Gomes, A. Carvalho, and A. H. Castro Neto, Phys. Rev. B 93, 045431 (2016).

[8] M. Mehboudi, B. M. Fregoso, Y. Yang, W. Zhu, A. van der Zande, J. Ferrer, L. Bellaiche, P. Kumar, and S. Barraza-Lopez, Phys. Rev. Lett. 117, 246802 (2016).

[9] L. Fu, Phys. Rev. Lett. 106, 106802 (2011).

[10] T. H. Hsieh, H. Lin, J. Liu, W. Duan, A. Bansil, and L. Fu, Nat. Commun. 3, 982 (2012).

[11] Y. Tanaka, Z. Ren, K. Nakayama, S. Souma, T. Takahashi, K. Segawa, and Y. Ando, Nat. Phys. 8, 800 (2012).

[12] Y. Sun, Z. Zhong, T. Shirakawa, C. Franchini, D. Li, Y. Li, S. Yunoki, and X.-Q. Chen, Phys. Rev. B 88, 235122 (2013).

[13] P. Dziawa, B. J. Kowalski, K. Dybko, R. Buczko, A. Szczerbakow, M. Szot, E. Łusakowska, T. Balasubramanian, B. M. Wojek, M. H. Berntsen, O. Tjernberg, and T. Story, Nat. Mater. 11, 1023 (2012).

[14] P. Barone, D. D. Sante, and S. Picozzi, Phys. Status Solidi RRL 7, 1102 (2013).

[15] P. Barone, T. Rauch, D. Di Sante, J. Henk, I. Mertig, and S. Picozzi, Phys. Rev. B 88, 045207 (2013).

[16] S. Safaei, M. Galicka, P. Kacman, and R. Buczko, New J. Phys. 17, 063041 (2015).

[17] J. Kim and S.-H. Jhi, Phys. Rev. B 92, 125142 (2015).

[18] J. Liu, X. Qian, and L. Fu, Nano Lett. 15, 2657 (2015).

[19] A. N. Mariano and K. L. Chopra, Appl. Phys. Lett. 10, 282 (1967).

[20] K. Momma and F. Izumi, J. Appl. Crystallogr. 44, 1272 (2011).

[21] I. Loa, R. J. Husband, R. A. Downie, S. R. Popuri, and J.-W. G. Bos, J. Phys.: Condens. Matter 27, 072202 (2015).

[22] X. Chen, P. Lu, X. Wang, Y. Zhou, C. An, Y. Zhou, C. Xian, H. Gao, Z. Guo, C. Park, B. Hou, K. Peng, X. Zhou, J. Sun, Y. Xiong, Z. Yang, D. Xing, and Y. Zhang, Phys. Rev. B 96, 165123 (2017).

[23] T. Chattopadhyay, A. Werner, H. von Schnering, and J. Pannetier, Rev. Phys. Appl. 19, 807 (1984).

[24] D. Zhou, Q. Li, W. Zheng, Y. Ma, and C. Chen, Phys. Chem. Chem. Phys. 19, 4560 (2017).

[25] D. Zhou, Q. Li, Y. Ma, Q. Cui, and C. Chen, J. Phys. Chem. C 117, 5352 (2013).

[26] H. Yu, W. Lao, L. Wang, K. Li, and Y. Chen, Phys. Rev. Lett. 118, 137002 (2017)

[27] Y. A. Timofeev, B. V. Vinogradov, and V. B. Begoulev, Phys. Solid State 39, 207 (1997).

[28] N. B. Kopnin, T. T. Heikkilä, and G. E. Volovik, Phys. Rev. B 83, 220503(R) (2011).

[29] R. Matsumoto, H. Hara, H. Tanaka, K. Nakamura, N. Kataoka, S. Yamamoto, A. Yamashita, S. Adachi, T. Irifune, H. Takeya, and Y. Takano, J. Phys. Soc. Jpn. 87, 124706 (2018).

[30] M. Kriener, M. Kamitani, T. Koretsune, R. Arita, Y. Taguchi, and Y. Tokura, Phys. Rev. Mater. 2, 044802 (2018).

[31] S. Gariglio, M. Gabay, and J.-M. Triscone, APL Mater. 4, 060701 (2016).

[32] N. Reyren, S. Thiel, A. D. Caviglia, L. F. Kourkoutis, G. Hammerl, C. Richter, C. W. Schneider, T. Kopp, A.-S. Ruetschi, D. Jaccard, M. Gabay, D. A. Muller, J.-M. Triscone, and J. Mannhart, Science 317, 1196 (2007).
[33] Y.-L. Han, S.-C. Shen, J. You, H.-O. Li, Z.-Z. Luo, C.-J. Li, G.-L. Qu, C.-M. Xiong, R.-F. Dou, L. He, D. Naugle, G.-P. Guo, and J.-C. Nie, Appl. Phys. Lett. 105, 192603 (2014).

[34] A. M. R. V. L. Monteiro, D. J. Groenendijk, I. Groen, J. de Bruijckere, R. Gaudenzi, H. S. J. van der Zant, and A. D. Caviglia, Phys. Rev. B 96, 020504 (2017).

[35] Y. Saito, T. Nojima, and Y. Iwasa, Nat. Rev. Mater. 2, 16094 (2016).

[36] J. M. Lu, O. Zheliuk, I. Leermakers, N. F. Q. Yuan, U. Zeitler, K. T. Law, and J. T. Ye, Science 350, 1353 (2015).

[37] A. W. Tsen, B. Hunt, Y. D. Kim, Z. J. Yuan, S. Jia, R. J. Cava, J. Hone, P. Kim, C. R. Dean, and A. N. Pasupathy, Nat. Phys. 12, 208 (2015).

[38] Y. Cao, V. Fatemi, S. Fang, K. Watanabe, T. Taniguchi, E. Kaxiras, and P. Jarillo-Herrero, Nature 556, 43 (2018).

[39] S. Caprara, M. Grilli, L. Benfatto, and C. Castellani, Phys. Rev. B 84, 014514 (2011).

[40] G. Dezi, N. Scopigno, S. Caprara, and M. Grilli, Phys. Rev. B 98, 214507 (2018)

[41] A. Larkin and A. Varlamov, Theory of Fluctuations in Superconductors, International series of Monographs on Physics (Clarendon Press, Oxford, 2005).

[42] P. Giannozzi, S. Baroni, N. Bonini, M. Calandra, R. Car, C. Cavazzoni, D. Ceresoli, G. L. Chiarotti, M. Cococcioni, I. Dabo, A. D. Corso, S. de Gironcoli, S. Fabris, G. Fratesi, R. Gebauer, U. Gerstmann, C. Gougoussis, A. Kokalj, M. Lazzeri, L. Martin-Samos, N. Marzari, F. Mauri, R. Mazzarello, S Paolini, A. Pasquarello, L. Paulatto, C. Sbraccia, S. Scandolo, G. Sclauzero, A. P. Seitsonen, A. Smogunov, P. Umari, and R. M. Wentzcovitch, J. Phys.: Condens. Matter 21, 395502 (2009).

[43] P. Giannozzi, O. Andreussi, T. Brumme, O. Bunau, M. B Nardelli, M. Calandra, R. Car, C. Cavazzoni, D. Ceresoli, M. Cococcioni, N. Colonna, I. Carnimeo, A. D. Corso, S. de Gironcoli, P. Delugas, R. A. DiStasio, A. Ferretti, A. Floris, G. Fratesi, G. Fugallo, R. Gebauer, U. Gerstmann, F. Giustino, T. Gorni, J. Jia, M. Kawamura, H.-Y. Ko, A. Kokalj, E. Küçükbenli, M. Lazzeri, M. Marsili, N. Marzari, F. Mauri, N. L. Nguyen, H.-V. Nguyen, A. O. de-la Roza, L. Paulatto, S. Poncé, D. Rocca, R. Sabatini, B. Santra, M. Schlipf, A. P. Seitsonen, A. Smogunov, I. Timrov, T. Thonhauser, P. Umari, N. Vast, X. Wu, and S. Baroni, J. Phys.: Condens. Matter 29, 465901 (2017).

[44] J. P. Perdew and A. Zunger, Phys. Rev. B 23, 5048 (1981).

[45] H. J. Monkhorst and J. D. Pack, Phys. Rev. B 13, 5188 (1976).

[46] F. D. Murnaghan, Proc. Nat. Acad. Sci. USA 30, 244 (1944).

[47] F. Birch, Phys. Rev. 71, 809 (1947).

[48] S. Baroni, P. Giannozzi, and A. Testa, Phys. Rev. Lett. 58, 1861 (1987).

[49] L. N. Oliveira, E. K. U. Gross, and W. Kohn, Phys. Rev. Lett. 60, 2430 (1988).

[50] M. Lüders, M. A. L. Marques, N. N. Lathiotakis, A. Floris, G. Profeta, L. Fast, A. Continenza, S. Massidda, and E. K. U. Gross, Phys. Rev. B 72, 024545 (2005).

[51] M. A. L. Marques, M. Lüders, N. N. Lathiotakis, G. Profeta, A. Floris, L. Fast, A. Continenza, E. K. U. Gross, and S. Massidda, Phys. Rev. B 72, 024546 (2005).

[52] A. Floris, G. Profeta, N. N. Lathiotakis, M. Lüders, M. A. L. Marques, C. Franchini, E. K. U. Gross, A. Continenza, and S. Massidda, Phys. Rev. Lett. 94, 037004 (2005). 
[53] P. Cudazzo, G. Profeta, A. Sanna, A. Floris, A. Continenza, S. Massidda, and E. K. U. Gross, Phys. Rev. Lett. 100, 257001 (2008).

[54] J. A. Flores-Livas, M. Amsler, C. Heil, A. Sanna, L. Boeri, G. Profeta, C. Wolverton, S. Goedecker, and E. K. U. Gross, Phys. Rev. B 93, 020508(R) (2016).

[55] A. Sanna, J. A. Flores-Livas, A. Davydov, G. Profeta, K. Dewhurst, S. Sharma, and E. K. U. Gross, J. Phys. Soc. Jpn. 87, 041012 (2018).

[56] K. Yokogawa, K. Murata, H. Yoshino, and S. Aoyama, Jpn. J. Appl. Phys. 46, 3636 (2007).

[57] M. S. Torikachvili, S. K. Kim, E. Colombier, S. L. Bud'ko, and P. C. Canfield, Rev. Sci. Instrum. 86, 123904 (2015).

[58] C. R. Rotundu, T. Ćuk, R. L. Greene, Z.-X. Shen, R. J. Hemley, and V. V. Struzhkin, Rev. Sci. Instrum. 84, 063903 (2013).

[59] D. Zhou, Q. Li, Y. Ma, Q. Cui, and C. Chen, J. Phys. Chem. C 117, 12266 (2013).

[60] A. Sanna, C. Franchini, A. Floris, G. Profeta, N. N. Lathiotakis, M. Lüders, M. A. L. Marques, E. K. U. Gross, A. Continenza, and S. Massidda, Phys. Rev. B 73, 144512 (2006).

[61] G. Profeta, C. Franchini, N. N. Lathiotakis, A. Floris, A. Sanna, M. A. L. Marques, M. Lüders, S. Massidda, E. K. U. Gross, and A. Continenza, Phys. Rev. Lett. 96, 047003 (2006).

[62] H. Suhl, B. T. Matthias, and L. R. Walker, Phys. Rev. Lett. 3, 552 (1959).
[63] S. Buga, V. Kulbachinskii, V. Kytin, G. Kytin, I. Kruglov, N. Lvova, N. Perov, N. Serebryanaya, S. Tarelkin, and V. Blank, Chem. Phys. Lett. 631-632, 97 (2015).

[64] H. Liu, Y. Li, G. Gao, J. S. Tse, and I. I. Naumov, J. Phys. Chem. C 120, 3458 (2016).

[65] K. Nguyen-Cong, J. M. Gonzalez, B. A. Steele, and I. I. Oleynik, J. Phys. Chem. C 122, 18274 (2018).

[66] R. Matsumoto, P. Song, S. Adachi, Y. Saito, H. Hara, A. Yamashita, K. Nakamura, S. Yamamoto, H. Tanaka, T. Irifune, H. Takeya, and Y. Takano, Phys. Rev. B 99, 184502 (2019).

[67] N. Colonna, G. Profeta, and A. Continenza, Phys. Rev. B 83, 224526 (2011).

[68] S. Caprara, D. Bucheli, N. Scopigno, N. Bergeal, J. Biscaras, S. Hurand, J. Lesueur, and M. Grilli, Supercond. Sci. Technol. 28, 014002 (2015).

[69] G. Venditti, J. Biscaras, S. Hurand, N. Bergeal, J. Lesueur, A. Dogra, R. C. Budhani, M. Mondal, J. Jesudasan, P. Raychaudhuri, S. Caprara, and L. Benfatto, Phys. Rev. B 100, 064506 (2019).

[70] S. Kirkpatrick, Rev. Mod. Phys. 45, 574 (1973).

[71] M. Monni, F. Bernardini, A. Sanna, G. Profeta, and S. Massidda, Phys. Rev. B 95, 064516 (2017).

[72] J. A. Flores-Livas, A. Sanna, A. P. Drozdov, L. Boeri, G. Profeta, M. Eremets, and S. Goedecker, Phys. Rev. Mater. 1, 024802 (2017). 